\title{
Intake of Potatoes Is Associated with Higher Diet Quality, and Improved Nutrient Intake and Adequacy among US Adolescents: NHANES 2001-2018 Analysis
}

\author{
Sanjiv Agarwal ${ }^{1, *(1)}$ and Victor L. Fulgoni, III ${ }^{2}$ (i) \\ 1 NutriScience LLC, East Norriton, PA 19403, USA \\ 2 Nutrition Impact, LLC, Battle Creek, MI 49014, USA; VIC3RD@aol.com \\ * Correspondence: agarwal47@yahoo.com; Tel.: +1-630-383-9359
}

Citation: Agarwal, S.; Fulgoni, V.L., III. Intake of Potatoes Is Associated with Higher Diet Quality,

and Improved Nutrient Intake and Adequacy among US Adolescents: NHANES 2001-2018 Analysis.

Nutrients 2021, 13, 2614.

https://doi.org/10.3390/nu13082614

Academic Editor: Rosa Casas

Received: 25 June 2021

Accepted: 26 July 2021

Published: 29 July 2021

Publisher's Note: MDPI stays neutral with regard to jurisdictional claims in published maps and institutional affiliations.

Copyright: () 2021 by the authors. Licensee MDPI, Basel, Switzerland. This article is an open access article distributed under the terms and conditions of the Creative Commons Attribution (CC BY) license (https:// creativecommons.org/licenses/by/ $4.0 /)$.

\begin{abstract}
Potatoes are nutrient rich white vegetables, however, research on their impact on public health is limited. The objective of this study was to provide updated evaluation of the cross-sectional association between potato consumption and diet quality, nutrient intake and adequacy. Twenty-four hour diet recall data from adolescents ( $n=16,633$; age 9-18 years) were used to assess intakes. Usual intakes of nutrients were determined using the National Cancer Institute method and diet quality was calculated using the Healthy Eating Index-2015 (HEI-2015) after adjusting for demographic factors. Consumers of potatoes (baked or boiled potatoes, mashed potatoes and potato mixtures, fried potatoes, and potato chips) had higher $(p<0.05)$ HEI-2015 total score and subcomponent scores for total vegetables, total protein foods, and refined grain than non-consumers. Consumers also had higher $(p<0.05)$ intake of energy, dietary fiber, protein, copper, magnesium, phosphorus, potassium, selenium, sodium, zinc, niacin, vitamin $\mathrm{B}_{6}$, vitamin $\mathrm{C}$, vitamin $\mathrm{K}$ and total choline; and higher $(p<0.05)$ adequacy for protein, copper, magnesium, phosphorus, potassium, zinc, thiamine, niacin, vitamin $\mathrm{B}_{6}$, vitamin $\mathrm{C}$, and vitamin $\mathrm{K}$ than non-consumers. In conclusion, adolescent potato consumption was associated with higher diet quality, nutrient intake, and adequacy and therefore encouraging their consumption may be an effective strategy for improving nutritional status.
\end{abstract}

Keywords: Healthy Eating Index; HEI; minerals; vitamins

\section{Introduction}

Potatoes are the most widely consumed non-cereal staple food consumed worldwide [1]. They are nutrient-rich, typically white, vegetables providing significant amounts of key essential nutrients, including dietary fiber, potassium, magnesium, vitamin C, vitamin B6, and phytonutrients [2-4] and are a more affordable source of nutrients with more favorable overall nutrient-to-price ratio compared to many other vegetables [5]. While potatoes are regarded as vegetables in United States (US) dietary recommendations [6], they are not considered as vegetables by World Health Organization [7] and are grouped as cereals in the United Kingdom National Food guide [8]. In the US, potato consumption is over 100 pounds per year per capita, accounting for $\sim 30 \%$ of total vegetable intake [9]. Their intake was estimated to be 0.35 cup equivalents/day/person in an analysis of National Health and Nutrition Examination Survey (NHANES) 2009-2010 [10].

Potatoes are classified as starchy vegetables because carbohydrates and especially starch (amylose, amylopectin, and resistant starch) are their predominant macronutrient. While potatoes are rich in carbohydrates (starch), because of their high amount of water they have a low energy density compared to other carbohydrate sources [11]. Cooking, cooling, and re-heating increases resistant starch levels in potatoes. Emerging research suggests that resistant starch may enhance satiety, may affect body composition, blood lipid and blood glucose levels; and stimulate growth and increase number of pro-bacteria in the colon [12,13]. 
Current scientific research on potato consumption and its impact on public health is controversial. In prospective studies, consumption of potatoes was associated with higher risk of type 2 diabetes and hypertension [14,15]. However, a systematic review published in 2016 did not find any conclusive evidence suggesting an association between potato intake and the risk of developing obesity and type 2 diabetes [16]. A recently published NHANES 1999-2010 data analysis reported positive association of higher potato intake with cardiometabolic risk factors but did not find any significant effects on long-term mortality rates [17]. However, only very limited data are available and a research gap exists in children and adolescents.

White potatoes represented about a third of all vegetable servings consumed by US children and adolescents in an analysis of NHANES 2003-2008 [18]. In another analysis of NHANES 2003-2006, white potatoes including French fries contributed to intake of energy and several shortfall nutrients in the diets of children and adolescents [19]. A dietary modeling analysis of NHANES 2005-2012 indicated that removing potatoes from children's diet might compromise potassium intake [20]. Federal nutrition policies related to the role of white potatoes in the diet of adolescents have been controversial and continuously evolving. In 2009, the Institute of Medicine (IOM) and United States Department of Agriculture (USDA) recommended to limit/excluded potatoes and other starchy vegetables from school meal plan [21,22]; however, in 2012, the proposal was struck down by the US Senate prohibiting USDA from setting any maximum limits on the serving of vegetables in school meal programs and removing the restriction on starchy vegetables (including French fries) in school lunches [22]. Starchy vegetables, including potatoes, were also initially restricted to one cup per week in the Special Supplemental Nutrition Program for Women, Infants, and Children (WIC) and the Health Hunger Free Kids Act [23] and in 2009 the WIC program offered a monthly fruit and vegetable cash value voucher that excluded white potatoes [24]; however, an IOM report in 2015 [25] indicated that white potatoes are particularly high in potassium and their increased consumption may help reduce shortfalls of potassium in the diets of both children and women, and recommended that they should be included as eligible vegetables under WIC program. In a recent analysis of longitudinal data from the National Heart, Lung, and Blood Institute's Growth and Health Study, intake of potassium, magnesium, vitamin B6, and vitamin C increased with increasing intake of potatoes at the baseline in adolescent girls [26].

Given this background, we hypothesize that potatoes as nutrient rich vegetables would be associated with improved diet quality and nutrient intake in all adolescents. Therefore, the purpose of this study was to provide an updated evaluation of the association of potato consumption among adolescents on diet quality, nutrient intake, and nutrient adequacy using NHANES 2001-2018 data. To separate the effects of less processed potatoes from more processed potatoes, we used three different definitions to define potato consumers-(1) those consuming potatoes as baked, boiled, and mashed and mixtures; (2) those consuming potatoes as baked, boiled, mashed and mixtures, and fried; and (3) those consuming potatoes as baked, boiled, mashed and mixtures, fried, and chips.

\section{Materials and Methods}

NHANES, a continuous large survey of a nationally representative sample of the non-institutionalized US population conducted by the National Center for Health Statistics, were used to assess potato intake [27]. $24 \mathrm{~h}$ dietary recall data of 16,633 adolescents 9-18 years participating in nine cycles of NHANES 2001-2018 were combined for the analyses to increase the sample size. Data from pregnant or lactating females and those with unreliable or incomplete data determined by the USDA were excluded. NHANES data are collected using a complex stratified multistage cluster sampling probability design. A detailed description of the subject recruitment, survey design, and data collection procedures is available online [27], and all data obtained for this study are publicly available at http:/ / www.cdc.gov/nchs/nhanes/, (accessed on 11 December 2020). All participants or proxies provided written informed consent and the Research Ethics Review Board at 
the National Center for Health Statistics approved the survey protocol. NHANES has stringent consent protocols and procedures to ensure confidentiality and protection from identification. This study was a secondary data analysis which lacked personal identifiers, therefore, did not require Institutional Review Board review.

Dietary intake of energy, nutrients, and food components was determined using 24-h dietary recalls, which included an in-person interview in the Mobile Examination Center (day 1 recall), followed by a telephone interview conducted 3-10 days after (day 2 recall). Parents or guardians assisted 9-11 years old adolescents, while 12-18 years old adolescents provided their own recalls. Participants were dichotomized into consumers and nonconsumers of potatoes; and consumers were further classified into intake quartiles based on individual usual intakes. Consumers were defined as those individuals consuming any amount of potatoes on either of the two days of dietary recall. Potato intakes were assessed using a total of four What We Eat in America (WWEIA) food categories (numbers indicated assigned by USDA for categorization purposes): baked or boiled potatoes (\#6802), mashed potatoes and potato mixtures (\#6806), fried potatoes (\#6804), and potato chips (\#5002). We used three definitions for defining potato intake:

Case 1: included WWEIA categories 6802 (baked or boiled), 6806 (mashed and mixtures)

Case 2: included WWEIA categories 6802, 6806, 6804 (fried)

Case 3: included WWEIA categories 6802, 6806, 6804, 5002 (chips)

Demographic information and physical activity levels were determined from the NHANES interview [27].

Diet quality scores were determined using the USDA Healthy Eating Index-2015 (HEI-2015) [28], which has 13 subcomponents, each reflecting an aspect of the 2015-2020 Dietary Guidelines for Americans [29] recommendations. Dietary intake was expressed per $1000 \mathrm{kcal}$ for all subcomponents, except for fatty acid ratios, which were expressed as ratio of unsaturated to saturated fatty acids, and for saturated fat and added sugars, both expressed as \% energy. Subcomponents for total vegetables; greens and beans; total fruit, whole fruit; total protein; and seafoods and plant proteins were scored proportionally from 0 to 5 points and all other subcomponents (i.e., whole grains; dairy; fatty acids; sodium; refined grains; saturated fat; and added sugars) were scored proportionally from 0 to 10 points. Four subcomponents, sodium, refined grains, saturated fat, and added sugars were reverse scored, so that lower intake leads to a higher score, and thus a greater contribution to overall diet quality. The maximum possible score was 100 [28]. The intake on the first dietary recall were used to determine HEI-2015 total and subcomponent scores.

Energy and nutrient intake were determined using the NHANES cycle specific USDA Food \& Nutrient Database for Dietary Studies [30,31]. Food components to assess HEI were determined using the NHANES cycle specific USDA MyPyramid Servings Database Food or Food Patterns Equivalents Database [32,33].

Analyses were performed using SAS 9.4 and data adjusted for the complex sampling (clustered sample) design of NHANES, using appropriate survey weights, strata, and primary sampling units. Least-square means (and the standard errors of the least-square means) for diet quality (HEI-2015 score), and for energy and nutrient intake were generated using regression analyses adjusted for key covariates (age, gender, and ethnicity). Usual intakes of energy, nutrients, and food groups were determined using the National Cancer Institute (NCI) method and NCI macros were used to estimate distribution of usual intake [34]. Nutrient adequacy was estimated by assessing the percentage of the population below the Estimated Average Requirement (EAR) or above Adequate Intake (AI) of nutrients using the cut-point method (except for iron, where the probability method was used, which is the recommended approach when assessing iron intake given asymmetrical requirement distribution of iron in menstruating women [35]). Significant differences between consumers and non-consumers were assessed via t-test and regression analyses were used to assess the linear relationship of potato intake quartiles with diet quality and nutrient intake. 


\section{Results}

Mean per capita intake of potatoes for Case 1 (baked, boiled, and mashed and mixtures), Case 2 (baked, boiled, mashed and mixtures, and fried), and Case 3 (baked, boiled, mashed and mixtures, fried, and chips) were $0.11 \pm 0.01,0.22 \pm 0.01$, and $0.32 \pm 0.01$ cup eq, respectively, for NHANES 2001-2018. Mean per capita intake of potatoes (for all three definitions) was stable and did not change significantly $(p>0.05)$ over the last 18 years (9 NHANES cycles) among US adolescents (Figure 1).

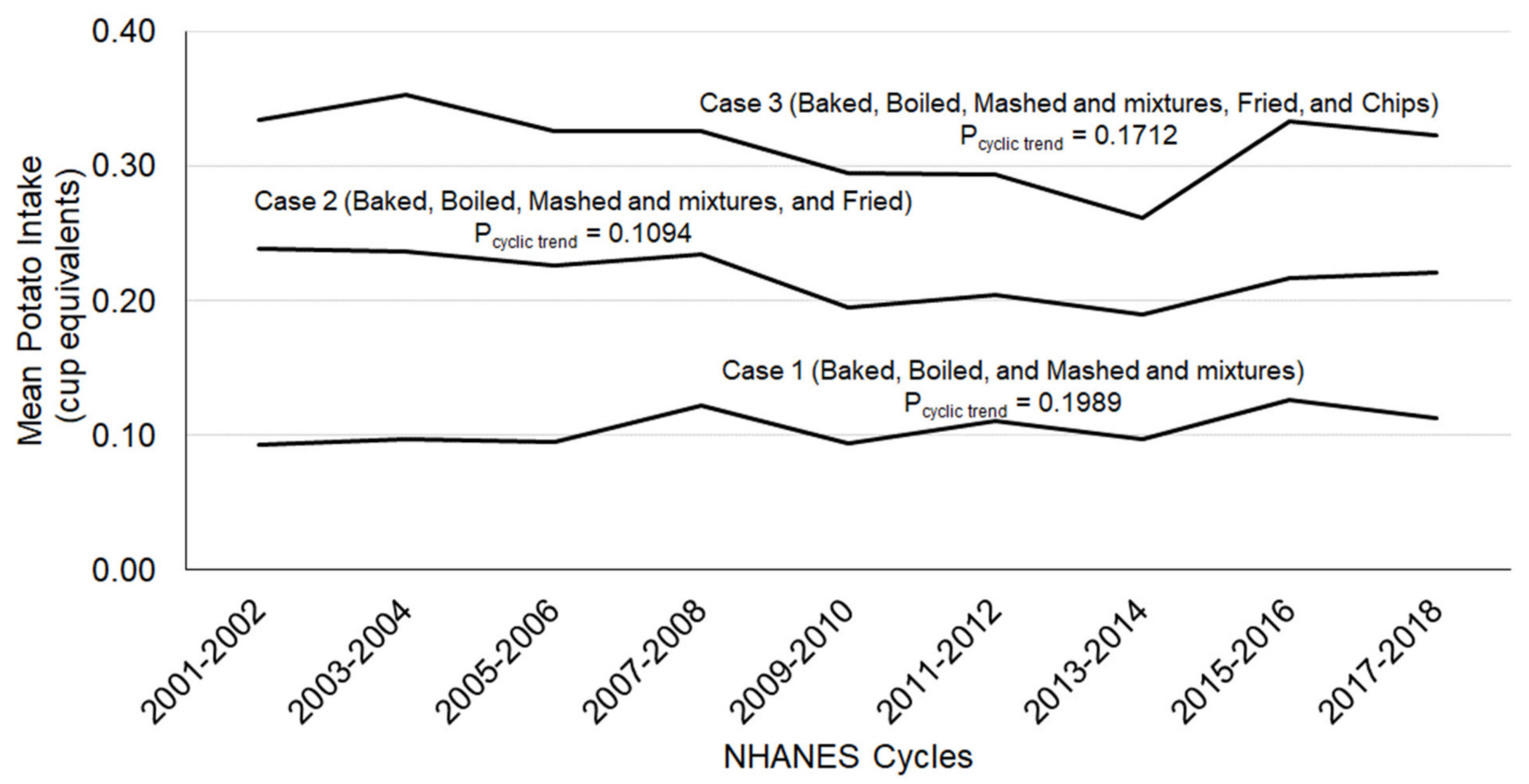

Figure 1. Covariate (age, gender, and ethnicity) adjusted mean intake of potatoes among adolescents (9-18 years of age, $n=16,633$ ) by NHANES study periods; gender combined data.

Demographics: Approximately $15.2 \%$ of adolescents were Case 1 potato (baked, boiled, mashed and mixtures) consumers. A lower $(\mathrm{p}<0.05)$ proportion of potato consumers were male $(-10.3 \%)$, Mexican American $(-18.3 \%)$, other Hispanic $(-28.4 \%)$, non-Hispanic Black $(-16.4 \%)$, other ethnicity $(-23.1 \%)$, and overweight $(-13.0 \%)$, while a greater proportion of Case 1 potato consumers were non-Hispanic White $(+16.1 \%)$ as compared to non-consumers (Table 1). All other demographic characteristics evaluated were similar $(p>0.05)$ among consumers and non-consumers potatoes in Case 1 (Table 1).

Table 1. Demographics associated with potato consumption in adolescents (9-18 years of age, $n=16,633)$, NHANES 2001-2018.

\begin{tabular}{|c|c|c|c|c|c|c|c|c|c|}
\hline & \multicolumn{3}{|c|}{$\begin{array}{c}\text { Case } 1 \text { (Baked, Boiled, } \\
\text { and Mashed and Mixtures) }\end{array}$} & \multicolumn{3}{|c|}{$\begin{array}{l}\text { Case } 2 \text { (Baked, Boiled, Mashed } \\
\text { and Mixtures, and Fried Potatoes) }\end{array}$} & \multicolumn{3}{|c|}{$\begin{array}{c}\text { Case } 3 \text { (Baked, Boiled, Mashed } \\
\text { and Mixtures, Fried Potatoes, } \\
\text { and Potato Chips) }\end{array}$} \\
\hline & $\begin{array}{l}\text { Non- } \\
\text { Consumer }\end{array}$ & Consumer & $p$ Value & $\begin{array}{c}\text { Non- } \\
\text { Consumer }\end{array}$ & Consumer & $p$ Value & $\begin{array}{l}\text { Non- } \\
\text { Consumer }\end{array}$ & Consumer & $p$ Value \\
\hline Sample N & 14,102 & 2531 & & 9455 & 7178 & & 7228 & 9405 & \\
\hline $\begin{array}{c}\text { Mean Age } \\
\text { (years) }\end{array}$ & $\begin{array}{c}13.6 \pm \\
0.04\end{array}$ & $13.4 \pm 0.1$ & 0.1289 & $\begin{array}{c}13.5 \pm \\
0.04\end{array}$ & $13.6 \pm 0.1$ & 0.5562 & $13.6 \pm 0.1$ & $13.5 \pm 0.1$ & 0.5546 \\
\hline $\begin{array}{c}\text { Gender (\% } \\
\text { Male) }\end{array}$ & $51.3 \pm 0.7$ & $46.0 \pm 1.4$ & 0.0006 & $52.2 \pm 0.9$ & $48.1 \pm 1.0$ & 0.0031 & $52.2 \pm 0.9$ & $49.0 \pm 0.9$ & 0.0192 \\
\hline $\begin{array}{l}\text { Ethnicity } \\
\text { Mexican } \\
\text { American (\%) }\end{array}$ & $14.2 \pm 0.9$ & $11.6 \pm 1.2$ & 0.0037 & $14.9 \pm 1.1$ & $12.3 \pm 0.8$ & 0.0021 & $15.5 \pm 1.1$ & $12.4 \pm 0.9$ & 0.0003 \\
\hline
\end{tabular}


Table 1. Cont.

\begin{tabular}{|c|c|c|c|c|c|c|c|c|c|}
\hline & \multicolumn{3}{|c|}{$\begin{array}{c}\text { Case } 1 \text { (Baked, Boiled, } \\
\text { and Mashed and Mixtures) }\end{array}$} & \multicolumn{3}{|c|}{$\begin{array}{c}\text { Case } 2 \text { (Baked, Boiled, Mashed } \\
\text { and Mixtures, and Fried Potatoes) }\end{array}$} & \multicolumn{3}{|c|}{$\begin{array}{c}\text { Case } 3 \text { (Baked, Boiled, Mashed } \\
\text { and Mixtures, Fried Potatoes, } \\
\text { and Potato Chips) }\end{array}$} \\
\hline & $\begin{array}{l}\text { Non- } \\
\text { Consumer }\end{array}$ & Consumer & $p$ Value & $\begin{array}{l}\text { Non- } \\
\text { Consumer }\end{array}$ & Consumer & $p$ Value & $\begin{array}{l}\text { Non- } \\
\text { Consumer }\end{array}$ & Consumer & $p$ Value \\
\hline $\begin{array}{c}\text { Other Hispanic } \\
(\%)\end{array}$ & $\begin{array}{c}6.69 \pm \\
0.58\end{array}$ & $\begin{array}{c}4.79 \pm \\
0.64\end{array}$ & 0.0036 & $\begin{array}{c}6.90 \pm \\
0.64\end{array}$ & $\begin{array}{c}5.64 \pm \\
0.56\end{array}$ & 0.0306 & $\begin{array}{c}7.17 \pm \\
0.68\end{array}$ & $\begin{array}{c}5.73 \pm \\
0.52\end{array}$ & 0.0106 \\
\hline $\begin{array}{l}\text { non-Hispanic } \\
\text { White }(\%)\end{array}$ & $56.0 \pm 1.4$ & $65.0 \pm 2.0$ & $<0.0001$ & $56.6 \pm 1.5$ & $58.9 \pm 1.6$ & 0.0760 & $56.7 \pm 1.6$ & $58.2 \pm 1.6$ & 0.2819 \\
\hline $\begin{array}{c}\text { non-Hispanic } \\
\text { Black }(\%)\end{array}$ & $14.6 \pm 0.8$ & $12.2 \pm 1.1$ & 0.0060 & $13.0 \pm 0.8$ & $15.8 \pm 1.0$ & 0.0001 & $11.8 \pm 0.8$ & $16.1 \pm 1.0$ & $<0.0001$ \\
\hline Other (\%) & $\begin{array}{c}8.45 \pm \\
0.51\end{array}$ & $\begin{array}{c}6.50 \pm \\
0.77\end{array}$ & 0.0130 & $\begin{array}{c}8.67 \pm \\
0.56\end{array}$ & $\begin{array}{c}7.37 \pm \\
0.56\end{array}$ & 0.0296 & $\begin{array}{c}8.78 \pm \\
0.63\end{array}$ & $7.59 \pm 0.5$ & 0.0459 \\
\hline \multicolumn{10}{|l|}{$\begin{array}{l}\text { Poverty Income } \\
\text { Ratio }\end{array}$} \\
\hline$<1.35(\%)$ & $32.2 \pm 1.1$ & $30.4 \pm 1.8$ & 0.3077 & $32.1 \pm 1.2$ & $31.6 \pm 1.2$ & 0.6925 & $31.9 \pm 1.3$ & $31.9 \pm 1.2$ & 0.9704 \\
\hline $1.35-1.85(\%)$ & $10.8 \pm 0.5$ & $11.3 \pm 1.0$ & 0.6185 & $10.8 \pm 0.6$ & $10.9 \pm 0.6$ & 0.9480 & $10.6 \pm 0.7$ & $11.1 \pm 0.6$ & 0.5288 \\
\hline $\begin{array}{l}>1.85(\%) \\
\text { Education }\end{array}$ & $57.0 \pm 1.2$ & $58.3 \pm 1.7$ & 0.4642 & $57.0 \pm 1.3$ & $57.5 \pm 1.3$ & 0.7167 & $57.5 \pm 1.4$ & $57.1 \pm 1.3$ & 0.7703 \\
\hline $\begin{array}{l}<\text { High School } \\
(\%)\end{array}$ & $98.6 \pm 0.2$ & $98.1 \pm 0.5$ & 0.3469 & $98.8 \pm 0.2$ & $98.1 \pm 0.3$ & 0.0204 & $98.9 \pm 0.2$ & $98.2 \pm 0.2$ & 0.0277 \\
\hline $\begin{array}{c}\text { High School < } \\
\text { Bachelor } \\
\text { Degree (\%) } \\
\text { Physical } \\
\text { Activity }\end{array}$ & $\begin{array}{c}1.42 \pm \\
0.16\end{array}$ & $\begin{array}{c}1.91 \pm \\
0.49\end{array}$ & 0.3469 & $\begin{array}{c}1.19 \pm \\
0.16\end{array}$ & $\begin{array}{c}1.92 \pm \\
0.27\end{array}$ & 0.0204 & $\begin{array}{c}1.15 \pm \\
0.17\end{array}$ & $\begin{array}{c}1.78 \pm \\
0.23\end{array}$ & 0.0277 \\
\hline Sedentary (\%) & $14.5 \pm 0.5$ & $14.2 \pm 1.0$ & 0.8119 & $14.4 \pm 0.6$ & $14.4 \pm 0.6$ & 0.9965 & $14.7 \pm 0.7$ & $14.2 \pm 0.6$ & 0.5265 \\
\hline Moderate (\%) & $24.6 \pm 0.6$ & $24.9 \pm 1.3$ & 0.8601 & $24.6 \pm 0.7$ & $24.7 \pm 0.8$ & 0.9820 & $24.4 \pm 0.9$ & $24.8 \pm 0.8$ & 0.7157 \\
\hline Vigorous (\%) & $60.9 \pm 0.7$ & $60.9 \pm 1.5$ & 0.9982 & $60.9 \pm 0.8$ & $60.9 \pm 0.9$ & 0.9809 & $60.9 \pm 1.0$ & $61.0 \pm 0.9$ & 0.9163 \\
\hline $\begin{array}{c}\text { Smoking Never } \\
(\%)\end{array}$ & $79.1 \pm 0.6$ & $82.3 \pm 1.7$ & 0.0702 & $79.9 \pm 0.8$ & $79.4 \pm 0.9$ & 0.6578 & $79.3 \pm 0.8$ & $79.9 \pm 0.8$ & 0.5726 \\
\hline $\begin{array}{l}\text { Smoking } \\
\text { Current }(\%)\end{array}$ & $\begin{array}{c}3.78 \pm \\
0.35\end{array}$ & $\begin{array}{c}3.01 \pm \\
0.60\end{array}$ & 0.2444 & $\begin{array}{c}3.88 \pm \\
0.42\end{array}$ & $\begin{array}{c}3.33 \pm \\
0.37\end{array}$ & 0.2695 & $\begin{array}{c}4.03 \pm \\
0.51\end{array}$ & $\begin{array}{c}3.34 \pm \\
0.34\end{array}$ & 0.2338 \\
\hline Overweight (\%) & $16.9 \pm 0.4$ & $14.7 \pm 1.0$ & 0.0439 & $16.7 \pm 0.5$ & $16.3 \pm 0.7$ & 0.6819 & $16.9 \pm 0.6$ & $16.2 \pm 0.6$ & 0.4541 \\
\hline Obese (\%) & $19.3 \pm 0.7$ & $18.3 \pm 1.0$ & 0.3711 & $18.4 \pm 0.7$ & $20.2 \pm 0.9$ & 0.0788 & $18.4 \pm 0.7$ & $19.8 \pm 0.8$ & 0.1729 \\
\hline
\end{tabular}

Data is presented as Mean \pm Standard Error. "Other Hispanic" and "Other" are not sampled in a way to be nationally representative.

Approximately $43.2 \%$ and $56.5 \%$ of adolescents were potato consumers by potato intake definition Case 2 (baked, boiled, mashed and mixtures, and fried) and Case 3 (baked, boiled, mashed and mixtures, fried, chips), respectively. For these potato definitions a lower $(p<0.05)$ proportion of adolescent consumers were male $(-7.85 \%$ and $-6.13 \%$, respectively), Mexican American ( $-17.4 \%$ and $-20.0 \%$, respectively), other Hispanic $(-18.3 \%$ and $-20.1 \%$, respectively), other ethnicity $(-15.0 \%$ and $-13.6 \%$ respectively), and below HS education $(-0.71 \%$ and $-0.71 \%$, respectively), and a higher $(p<0.05)$ proportion were $(p<0.05)$ non-Hispanic Black $(+21.5 \%$ and $+36.4 \%$, respectively) and had a HS education $(+61.3 \%$ and $+54.8 \%$, respectively). All other demographic characteristics evaluated were similar $(p>0.05)$ among consumers and non-consumers of potatoes in Case 2 and 3 (Table 1).

Diet Quality: Adolescent consumers of potatoes in Case 1 (baked, boiled, mashed and mixtures) as compared to non-consumers had higher HEI- 2015 total score $(+4.70 \%$, $p<0.01)$, and the score gradually increased with increasing intake quartile $(\beta=0.77 \pm 0.15$, $\left.\mathrm{P}_{\text {quartile trend }}<0.01\right)$. HEI-2015 subcomponent scores among potato consumers compared to non-consumers were also higher $(p<0.05)$ for total vegetables $(+49.5 \%)$, total protein foods $(+9.22 \%)$, refined grain $(+24.2 \%)$, and added sugar $(+5.76 \%)$; and gradually increased with increasing intake quartiles $(\beta=0.39 \pm 0.02, \beta=0.09 \pm 0.02, \beta=0.42 \pm 0.05, \beta=0.13$ \pm 0.04 , respectively; $\mathrm{P}_{\text {quartile trend }}<0.01$ for all). However, subcomponent scores among 
Case 1 potato consumers compared to non-consumers were lower for dairy and sodium $(-5.61 \%,-10.1 \%$, respectively; $p<0.01$ for both) and gradually decreased with increasing intake quartiles $\left(\beta=-0.15 \pm 0.03, \beta=-0.17 \pm 0.03\right.$, respectively; $\mathrm{P}_{\text {quartile trend }}<0.01$ for both) (Table 2).

Table 2. Healthy Eating Index (HEI) 2015 and sub-component scores in adolescents (9-18 years of age, $n=16,633$ ) potato (Case 1; baked, boiled, mashed and mixtures) consumers and non-consumers, and by intake quartiles, NHANES 2001-2018; gender combined data.

\begin{tabular}{|c|c|c|c|c|c|c|c|c|}
\hline \multirow[b]{2}{*}{$\begin{array}{l}\text { HEI } 2015 \\
\text { Components }\end{array}$} & \multirow[b]{2}{*}{$\begin{array}{c}\text { Non- } \\
\text { Consumers }\end{array}$} & \multirow[b]{2}{*}{ Consumers } & \multirow[b]{2}{*}{$p$ Value } & \multicolumn{5}{|c|}{ Potato Intake Quartiles for Consumers (Cup Equivalents) } \\
\hline & & & & $\begin{array}{c}\mathrm{Q1} \\
(<0.50)\end{array}$ & $\begin{array}{c}\mathrm{Q} 2 \\
(0.50 \text { to } \\
<0.59)\end{array}$ & $\begin{array}{c}\text { Q3 } \\
(0.59 \text { to } \\
<0.69)\end{array}$ & $\begin{array}{c}\mathrm{Q4} \\
(\geq 0.69)\end{array}$ & $\mathbf{P}_{\text {quartile trend }}$ \\
\hline Total score & $4.7 \pm 0.2$ & $46.8 \pm 0.4$ & $<0.0001$ & $46.8 \pm 0.7$ & $45.3 \pm 0.7$ & $46.5 \pm 0.7$ & $48.4 \pm 0.8$ & $<0.0001$ \\
\hline $\begin{array}{c}\text { Component } 1 \\
\text { (total vegetables) }\end{array}$ & $2.06 \pm 0.02$ & $3.08 \pm 0.04$ & $<0.0001$ & $\begin{array}{c}2.60 \pm \\
0.09\end{array}$ & $\begin{array}{c}2.94 \pm \\
0.07\end{array}$ & $\begin{array}{c}3.26 \pm \\
0.09\end{array}$ & $\begin{array}{c}3.52 \pm \\
0.11\end{array}$ & $<0.0001$ \\
\hline $\begin{array}{l}\text { Component } 2 \\
\text { (greens and beans) }\end{array}$ & $0.88 \pm 0.03$ & $0.85 \pm 0.05$ & 0.6393 & $\begin{array}{c}1.02 \pm \\
0.10\end{array}$ & $\begin{array}{c}0.75 \pm \\
0.11\end{array}$ & $\begin{array}{c}0.83 \pm \\
0.11\end{array}$ & $\begin{array}{c}0.78 \pm \\
0.10\end{array}$ & 0.3384 \\
\hline $\begin{array}{l}\text { Component } 3 \\
\text { (total fruit) }\end{array}$ & $2.07 \pm 0.04$ & $2.11 \pm 0.06$ & 0.5131 & $\begin{array}{c}2.05 \pm \\
0.13\end{array}$ & $\begin{array}{c}1.89 \pm \\
0.13\end{array}$ & $\begin{array}{c}2.09 \pm \\
0.12\end{array}$ & $\begin{array}{c}2.39 \pm \\
0.13\end{array}$ & 0.1010 \\
\hline $\begin{array}{l}\text { Component } 4 \\
\text { (whole fruit) }\end{array}$ & $1.82 \pm 0.04$ & $1.85 \pm 0.06$ & 0.6642 & $\begin{array}{c}1.86 \pm \\
0.14\end{array}$ & $\begin{array}{c}1.48 \pm \\
0.14\end{array}$ & $\begin{array}{c}1.81 \pm \\
0.13\end{array}$ & $\begin{array}{c}2.26 \pm \\
0.15\end{array}$ & 0.1095 \\
\hline $\begin{array}{l}\text { Component } 5 \\
\text { (whole grains) }\end{array}$ & $2.09 \pm 0.04$ & $2.12 \pm 0.10$ & 0.7451 & $\begin{array}{c}2.16 \pm \\
0.20\end{array}$ & $\begin{array}{c}1.95 \pm \\
0.18\end{array}$ & $\begin{array}{c}2.04 \pm \\
0.19\end{array}$ & $\begin{array}{c}2.33 \pm \\
0.20\end{array}$ & 0.5762 \\
\hline Component 6 (dairy) & $6.42 \pm 0.05$ & $6.06 \pm 0.10$ & 0.0001 & $\begin{array}{c}6.08 \pm \\
0.18\end{array}$ & $\begin{array}{c}6.57 \pm \\
0.19\end{array}$ & $\begin{array}{c}5.82 \pm \\
0.21\end{array}$ & $\begin{array}{c}5.76 \pm \\
0.22\end{array}$ & $<0.0001$ \\
\hline $\begin{array}{c}\text { Component } 7 \\
\text { (total protein foods) }\end{array}$ & $3.58 \pm 0.03$ & $3.91 \pm 0.05$ & $<0.0001$ & $\begin{array}{c}4.04 \pm \\
0.08\end{array}$ & $\begin{array}{c}3.93 \pm \\
0.07\end{array}$ & $\begin{array}{c}3.81 \pm \\
0.10\end{array}$ & $\begin{array}{c}3.85 \pm \\
0.11\end{array}$ & $<0.0001$ \\
\hline $\begin{array}{l}\text { Component } 8 \text { (seafood } \\
\text { and plant protein) }\end{array}$ & $1.53 \pm 0.03$ & $1.51 \pm 0.07$ & 0.8394 & $\begin{array}{c}1.57 \pm \\
0.11\end{array}$ & $\begin{array}{c}1.42 \pm \\
0.11\end{array}$ & $\begin{array}{c}1.44 \pm \\
0.12\end{array}$ & $\begin{array}{c}1.62 \pm \\
0.17\end{array}$ & 0.9606 \\
\hline $\begin{array}{c}\text { Component } 9 \\
\text { (fatty acid ratio) }\end{array}$ & $4.09 \pm 0.05$ & $4.05 \pm 0.13$ & 0.7803 & $\begin{array}{c}4.37 \pm \\
0.23\end{array}$ & $\begin{array}{c}3.85 \pm \\
0.22\end{array}$ & $\begin{array}{c}4.00 \pm \\
0.21\end{array}$ & $\begin{array}{c}4.00 \pm \\
0.25\end{array}$ & 0.5025 \\
\hline $\begin{array}{l}\text { Component } 10 \\
\text { (sodium) }\end{array}$ & $4.75 \pm 0.05$ & $4.27 \pm 0.10$ & $<0.0001$ & $\begin{array}{c}4.45 \pm \\
0.19\end{array}$ & $\begin{array}{c}4.18 \pm \\
0.21\end{array}$ & $\begin{array}{c}4.41 \pm \\
0.19\end{array}$ & $\begin{array}{c}4.05 \pm \\
0.19\end{array}$ & $<0.0001$ \\
\hline $\begin{array}{l}\text { Component } 11 \\
\text { (refined grain) }\end{array}$ & $4.72 \pm 0.05$ & $5.86 \pm 0.11$ & $<0.0001$ & $\begin{array}{c}5.55 \pm \\
0.23\end{array}$ & $\begin{array}{c}5.45 \pm \\
0.23\end{array}$ & $\begin{array}{c}6.27 \pm \\
0.19\end{array}$ & $\begin{array}{c}6.16 \pm \\
0.25\end{array}$ & $<0.0001$ \\
\hline $\begin{array}{l}\text { Component } 12 \\
\text { (saturated fat) }\end{array}$ & $5.54 \pm 0.05$ & $5.60 \pm 0.11$ & 0.6196 & $\begin{array}{c}5.75 \pm \\
0.23\end{array}$ & $\begin{array}{c}5.46 \pm \\
0.21\end{array}$ & $\begin{array}{c}5.54 \pm \\
0.18\end{array}$ & $\begin{array}{c}5.63 \pm \\
0.24\end{array}$ & 0.7499 \\
\hline $\begin{array}{l}\text { Component } 13 \\
\text { (added sugar) }\end{array}$ & $5.21 \pm 0.05$ & $5.51 \pm 0.12$ & 0.0177 & $\begin{array}{c}5.30 \pm \\
0.19\end{array}$ & $\begin{array}{c}5.48 \pm \\
0.21\end{array}$ & $\begin{array}{c}5.19 \pm \\
0.22\end{array}$ & $\begin{array}{c}6.04 \pm \\
0.19\end{array}$ & 0.0031 \\
\hline
\end{tabular}

Data adjusted for age, gender, and ethnicity; and presented as Least Square Mean \pm Standard Error.

Adolescent consumers of potatoes in Case 2 (baked, boiled, mashed, and mixtures, fried) as compared to non-consumers also had a higher $(2.01 \%, p<0.01)$ HEI-2015 total score, and higher $(p<0.01)$ subcomponent scores for total vegetables $(36.8 \%)$, total protein foods $(7.39 \%)$, fatty acid ratio $(16.5 \%)$, and refined grain $(27.5 \%)$. However, subcomponent scores among Case 2 potato consumers compared to non-consumers were lower $(p<0.01)$ for greens and beans $(-20.0 \%)$, total fruit $(-11.5 \%)$, whole fruit $(-13.4 \%)$, whole grain $(-18.5 \%)$, dairy $(-8.64 \%)$, and seafood and plant protein $(-11.9 \%)$ (Table 3$)$. 
Table 3. Healthy Eating Index (HEI) 2015 and sub-component scores associated with potato consumption in adolescents (9-18 years of age, $n=16,633)$-NHANES 2001-2018; gender combined data.

\begin{tabular}{|c|c|c|c|c|c|c|}
\hline \multirow[t]{2}{*}{ HEI 2015 Components } & \multicolumn{3}{|c|}{$\begin{array}{l}\text { Case } 2 \text { (Baked, Boiled, Mashed and } \\
\text { Mixtures, and Fried Potatoes) }\end{array}$} & \multicolumn{3}{|c|}{$\begin{array}{c}\text { Case } 3 \text { (Baked, Boiled, Mashed and } \\
\text { Mixtures, Fried Potatoes and } \\
\text { Potato Chips) }\end{array}$} \\
\hline & $\begin{array}{l}\text { Non- } \\
\text { Consumers }\end{array}$ & Consumers & $p$ Value & $\begin{array}{l}\text { Non- } \\
\text { Consumers }\end{array}$ & Consumers & $p$ Value \\
\hline Total score & $44.7 \pm 0.2$ & $45.6 \pm 0.2$ & 0.0062 & $44.7 \pm 0.3$ & $45.4 \pm 0.2$ & 0.0346 \\
\hline Component 1 (total vegetables) & $1.93 \pm 0.03$ & $2.64 \pm 0.03$ & $<0.0001$ & $1.81 \pm 0.03$ & $2.57 \pm 0.02$ & $<0.0001$ \\
\hline Component 2 (greens and beans) & $0.95 \pm 0.03$ & $0.76 \pm 0.04$ & $<0.0001$ & $1.02 \pm 0.04$ & $0.75 \pm 0.03$ & $<0.0001$ \\
\hline Component 3 (total fruit) & $2.18 \pm 0.04$ & $1.93 \pm 0.04$ & $<0.0001$ & $2.18 \pm 0.05$ & $1.99 \pm 0.04$ & 0.0012 \\
\hline Component 4 (whole fruit) & $1.94 \pm 0.05$ & $1.68 \pm 0.05$ & $<0.0001$ & $1.96 \pm 0.05$ & $1.73 \pm 0.05$ & 0.0002 \\
\hline Component 5 (whole grains) & $2.27 \pm 0.05$ & $1.85 \pm 0.06$ & $<0.0001$ & $2.39 \pm 0.06$ & $1.86 \pm 0.05$ & $<0.0001$ \\
\hline Component 6 (dairy) & $6.60 \pm 0.06$ & $6.03 \pm 0.07$ & $<0.0001$ & $6.69 \pm 0.07$ & $6.09 \pm 0.06$ & $<0.0001$ \\
\hline Component 7 (total protein foods) & $3.52 \pm 0.04$ & $3.78 \pm 0.03$ & $<0.0001$ & $3.53 \pm 0.04$ & $3.72 \pm 0.03$ & 0.0002 \\
\hline Component 8 (seafood and plant protein) & $1.60 \pm 0.03$ & $1.41 \pm 0.05$ & 0.0004 & $1.62 \pm 0.04$ & $1.44 \pm 0.04$ & 0.0007 \\
\hline Component 9 (fatty acid ratio) & $3.81 \pm 0.05$ & $4.44 \pm 0.08$ & $<0.0001$ & $3.70 \pm 0.06$ & $4.38 \pm 0.07$ & $<0.0001$ \\
\hline Component 10 (sodium) & $4.64 \pm 0.06$ & $4.70 \pm 0.07$ & 0.4611 & $4.59 \pm 0.07$ & $4.73 \pm 0.06$ & 0.0882 \\
\hline Component 11 (refined grain) & $4.40 \pm 0.06$ & $5.61 \pm 0.07$ & $<0.0001$ & $4.27 \pm 0.07$ & $5.42 \pm 0.06$ & $<0.0001$ \\
\hline Component 12 (saturated fat) & $5.57 \pm 0.05$ & $5.53 \pm 0.07$ & 0.5763 & $5.55 \pm 0.06$ & $5.55 \pm 0.06$ & 0.9781 \\
\hline Component 13 (added sugar) & $5.33 \pm 0.07$ & $5.18 \pm 0.07$ & 0.1064 & $5.41 \pm 0.07$ & $5.15 \pm 0.07$ & 0.0073 \\
\hline
\end{tabular}

Data adjusted for age, gender and ethnicity; and presented as Least Square Mean \pm Standard Error.

Adolescent consumers of potatoes in Case 3 (baked, boiled, mashed and mixtures, fried, chips) as compared to non-consumers had a higher $(1.57 \%, p<0.05)$ HEI-2015 total score, and higher $(p<0.01)$ subcomponent scores for total vegetables $(41.2 \%)$, total protein foods $(5.38 \%)$, fatty acid ratio $(18.4 \%)$ and refined grain $(26.9 \%)$; and lower $(p<0.01)$ subcomponent score for greens and beans $(-26.5 \%)$, total fruit $(-8.72 \%)$, whole fruit $(-11.7 \%)$, whole grain $(-22.2 \%)$, dairy $(-8.97 \%)$, seafood and plant protein $(-11.1 \%)$, added sugar $(-4.81 \%)$ (Table 3$)$.

Nutrient Intake: Adolescent consumers of potatoes in Case 1 (baked, boiled, mashed and mixtures) as compared to non-consumers had higher $(p<0.05)$ intake of energy $(+6.26 \%)$, carbohydrate $(+5.43 \%)$, dietary fiber $(+8.51 \%)$, protein $(+12.0 \%)$, copper $(+12.4 \%)$, magnesium $(+10.3 \%)$, phosphorus $(+6.62 \%)$, potassium $(+18.7 \%)$, selenium $(+6.86 \%)$, sodium $(+8.98 \%)$, zinc $(+9.09 \%)$, vitamin A $(+9.59 \%)$, thiamine $(+4.24 \%)$, niacin $(+11.7 \%)$, vitamin $\mathrm{B}_{6}(+20.8 \%)$, vitamin $\mathrm{C}(+23.1 \%)$, vitamin $\mathrm{K}(+22.2 \%)$, and total choline $(+14.9 \%)$. Intakes of all other nutrients evaluated were similar $(p>0.05)$ among consumers and non-consumers (Table 4).

Table 4. Covariate (age, gender, and ethnicity) adjusted energy and nutrient intakes in adolescents (9-18 years of age, $n=$ 16,633) potato (Case 1; baked, boiled, mashed and mixtures) consumers and non-consumers, by intake quartiles, NHANES 2001-2018; gender combined data.

\begin{tabular}{|c|c|c|c|c|c|c|c|c|}
\hline & \multirow[b]{2}{*}{$\begin{array}{c}\text { Non- } \\
\text { Consumers }\end{array}$} & \multirow[b]{2}{*}{ Consumers } & \multirow[b]{2}{*}{$p$ Value } & \multicolumn{5}{|c|}{ Potato Intake Quartiles for Consumers (Cup Equivalents) } \\
\hline & & & & $\begin{array}{c}\mathrm{Q1} \\
(<0.50)\end{array}$ & $\begin{array}{c}\mathrm{Q} 2 \\
(0.50 \text { to } \\
<0.59)\end{array}$ & $\begin{array}{c}\text { Q3 } \\
(0.59 \text { to } \\
<0.69)\end{array}$ & $\begin{array}{c}\mathrm{Q} 4 \\
\mathbf{(} \geq \mathbf{0 . 6 9 )}\end{array}$ & $\mathbf{P}_{\text {quartile trend }}$ \\
\hline Energy (kcal) & $2094 \pm 12$ & $2225 \pm 24$ & $<0.0001$ & $2187 \pm 41$ & $2189 \pm 49$ & $2157 \pm 48$ & $2366 \pm 61$ & $<0.0001$ \\
\hline Carbohydrate (gm) & $276 \pm 2$ & $291 \pm 3$ & 0.0002 & $286 \pm 6$ & $286 \pm 7$ & $283 \pm 7$ & $308 \pm 8$ & 0.0001 \\
\hline Dietary fiber $(\mathrm{gm})$ & $14.1 \pm 0.1$ & $15.3 \pm 0.2$ & $<0.0001$ & $14.6 \pm 0.4$ & $14.5 \pm 0.4$ & $14.5 \pm 0.4$ & $17.5 \pm 0.5$ & $<0.0001$ \\
\hline Protein $(\mathrm{gm})$ & $73.9 \pm 0.5$ & $82.8 \pm 1.3$ & $<0.0001$ & $80.4 \pm 1.8$ & $82.1 \pm 2.2$ & $77.9 \pm 2.0$ & $90.7 \pm 3.0$ & $<0.0001$ \\
\hline
\end{tabular}


Table 4. Cont.

\begin{tabular}{|c|c|c|c|c|c|c|c|c|}
\hline Calcium (mg) & $1029 \pm 10$ & $1023 \pm 18$ & 0.7320 & $1001 \pm 31$ & $1052 \pm 33$ & $960 \pm 34$ & $1080 \pm 40$ & 0.9113 \\
\hline Copper (mg) & $1.05 \pm 0.01$ & $1.18 \pm 0.02$ & $<0.0001$ & $\begin{array}{c}1.10 \pm \\
0.03\end{array}$ & $\begin{array}{c}1.12 \pm \\
0.04\end{array}$ & $1.15 \pm 0.03$ & $\begin{array}{c}1.35 \pm \\
0.05\end{array}$ & $<0.0001$ \\
\hline Iron (mg) & $15.2 \pm 0.1$ & $15.5 \pm 0.2$ & 0.2581 & $15.3 \pm 0.4$ & $15.5 \pm 0.4$ & $14.8 \pm 0.5$ & $16.5 \pm 0.5$ & 0.1380 \\
\hline Magnesium (mg) & $242 \pm 2$ & $267 \pm 4$ & $<0.0001$ & $253 \pm 6$ & $259 \pm 6$ & $254 \pm 6$ & $302 \pm 10$ & $<0.0001$ \\
\hline Phosphorus (mg) & $1315 \pm 9$ & $1402 \pm 19$ & $<0.0001$ & $1372 \pm 30$ & $1405 \pm 34$ & $1327 \pm 36$ & $1502 \pm 44$ & $<0.0001$ \\
\hline Potassium (mg) & $2205 \pm 17$ & $2617 \pm 34$ & $<0.0001$ & $2382 \pm 50$ & $2471 \pm 58$ & $2521 \pm 57$ & $3086 \pm 82$ & $<0.0001$ \\
\hline Selenium ( $\mu \mathrm{g})$ & $102 \pm 1$ & $109 \pm 2$ & 0.0003 & $108 \pm 3$ & $109 \pm 3$ & $101 \pm 3$ & $119 \pm 4$ & 0.0004 \\
\hline Sodium (mg) & $3363 \pm 26$ & $3665 \pm 48$ & $<0.0001$ & $3578 \pm 80$ & $\begin{array}{c}3625 \pm \\
103\end{array}$ & $3493 \pm 72$ & $\begin{array}{l}3959 \pm \\
116\end{array}$ & $<0.0001$ \\
\hline Zinc (mg) & $11.0 \pm 0.1$ & $12.0 \pm 0.2$ & $<0.0001$ & $11.3 \pm 0.3$ & $11.8 \pm 0.3$ & $11.6 \pm 0.4$ & $13.3 \pm 0.5$ & $<0.0001$ \\
\hline Vitamin A (RE) $(\mu \mathrm{g})$ & $584 \pm 7$ & $640 \pm 17$ & 0.0013 & $610 \pm 22$ & $632 \pm 33$ & $605 \pm 29$ & $711 \pm 47$ & 0.0017 \\
\hline Vitamin $\mathrm{B}_{6}(\mathrm{mg})$ & $1.78 \pm 0.02$ & $2.15 \pm 0.03$ & $<0.0001$ & $\begin{array}{c}1.96 \pm \\
0.05\end{array}$ & $\begin{array}{c}2.04 \pm \\
0.07\end{array}$ & $2.07 \pm 0.06$ & $\begin{array}{c}2.53 \pm \\
0.09\end{array}$ & $<0.0001$ \\
\hline Vitamin $\mathrm{B}_{12}(\mu \mathrm{g})$ & $5.12 \pm 0.07$ & $5.30 \pm 0.11$ & 0.1633 & $\begin{array}{c}5.13 \pm \\
0.17\end{array}$ & $\begin{array}{c}5.09 \pm \\
0.18\end{array}$ & $4.96 \pm 0.20$ & $\begin{array}{c}6.01 \pm \\
0.33\end{array}$ & 0.0515 \\
\hline Thiamin (mg) & $1.65 \pm 0.01$ & $1.72 \pm 0.03$ & 0.0126 & $\begin{array}{c}1.67 \pm \\
0.04\end{array}$ & $\begin{array}{c}1.72 \pm \\
0.05\end{array}$ & $1.63 \pm 0.05$ & $\begin{array}{c}1.87 \pm \\
0.06\end{array}$ & 0.0046 \\
\hline Riboflavin (mg) & $2.08 \pm 0.02$ & $2.15 \pm 0.03$ & 0.0690 & $\begin{array}{c}2.13 \pm \\
0.05\end{array}$ & $\begin{array}{c}2.15 \pm \\
0.06\end{array}$ & $2.01 \pm 0.06$ & $\begin{array}{c}2.29 \pm \\
0.08\end{array}$ & 0.0483 \\
\hline Niacin (mg) & $23.0 \pm 0.2$ & $25.7 \pm 0.4$ & $<0.0001$ & $24.7 \pm 0.6$ & $25.2 \pm 0.9$ & $24.4 \pm 0.7$ & $28.6 \pm 1.1$ & $<0.0001$ \\
\hline Folate, DFE $(\mu \mathrm{g})$ & $557 \pm 6$ & $554 \pm 14$ & 0.8296 & $550 \pm 19$ & $588 \pm 36$ & $527 \pm 22$ & $549 \pm 24$ & 0.6519 \\
\hline Vitamin C (mg) & $73.9 \pm 1.4$ & $91.0 \pm 3.6$ & $<0.0001$ & $78.6 \pm 4.3$ & $85.0 \pm 5.2$ & $90.4 \pm 4.9$ & $\begin{array}{c}109.6 \pm \\
11.6\end{array}$ & $<0.0001$ \\
\hline $\begin{array}{c}\text { Vitamin D (D2 + D3) } \\
(\mu \mathrm{g})\end{array}$ & $5.33 \pm 0.08$ & $5.52 \pm 0.15$ & 0.2182 & $\begin{array}{c}5.33 \pm \\
0.25\end{array}$ & $\begin{array}{c}5.62 \pm \\
0.30\end{array}$ & $5.08 \pm 0.25$ & $\begin{array}{c}6.06 \pm \\
0.36\end{array}$ & 0.1189 \\
\hline $\begin{array}{c}\text { Vitamin E (ATE) } \\
(\mathrm{mg})\end{array}$ & $7.10 \pm 0.10$ & $7.45 \pm 0.17$ & 0.0834 & $\begin{array}{c}7.50 \pm \\
0.35\end{array}$ & $\begin{array}{c}6.81 \pm \\
0.35\end{array}$ & $7.27 \pm 0.37$ & $\begin{array}{c}8.21 \pm \\
0.44\end{array}$ & 0.0437 \\
\hline Vitamin K $(\mu \mathrm{g})$ & $64.9 \pm 1.3$ & $79.3 \pm 3.7$ & 0.0003 & $81.0 \pm 7.4$ & $61.2 \pm 3.4$ & $84.5 \pm 10.8$ & $90.2 \pm 7.3$ & 0.0002 \\
\hline Total choline (mg) & $262 \pm 3$ & $301 \pm 6$ & $<0.0001$ & $292 \pm 10$ & $293 \pm 8$ & $272 \pm 8$ & $343 \pm 14$ & $<0.0001$ \\
\hline
\end{tabular}

Data presented as Least Square Mean \pm Standard Error. RE: retinol equivalent; DFE: dietary folate equivalent; ATE: alpha tocopherol equivalent.

Increasing intake quartiles also gradually increased $\left(\mathrm{P}_{\text {quartile trend }}<0.05\right)$ intake of energy $(\beta=51.9 \pm 10.2 \mathrm{kcal})$, carbohydrate $(\beta=5.86 \pm 1.47 \mathrm{mg})$, dietary fiber $(\beta=0.55 \pm$ $0.09 \mathrm{~g})$, protein $(\beta=3.36 \pm 0.50 \mathrm{~g})$, copper $(\beta=0.06 \pm 0.01 \mathrm{mg})$, magnesium $(\beta=10.6 \pm 1.6$ $\mathrm{mg})$, phosphorus $(\beta=33.8 \pm 7.2 \mathrm{mg})$, potassium $(\beta=174 \pm 12 \mathrm{mg})$, selenium $(\beta=2.75 \pm$ $0.75 \mu \mathrm{g})$, sodium $(\beta=116 \pm 18 \mathrm{mg})$, zinc $(\beta=0.43 \pm 0.08 \mathrm{mg})$, vitamin $\mathrm{A}(\beta=23.2 \pm 7.2)$, thiamine $(\beta=0.03 \pm 0.01 \mathrm{mg})$, riboflavin $(\beta=0.03 \pm 0.01 \mathrm{mg})$, niacin $(\beta=1.08 \pm 0.18 \mathrm{mg})$, vitamin $\mathrm{B}_{6}(\beta=0.15 \pm 0.01 \mathrm{mg})$, vitamin $\mathrm{C}(\beta=7.36 \pm 1.73 \mathrm{mg})$, vitamin $\mathrm{E}(\beta=0.16 \pm 0.08$ $\mathrm{mg})$, vitamin $\mathrm{K}(\beta=5.62 \pm 1.46 \mu \mathrm{g})$, and total choline $(\beta=15.1 \pm 2.2 \mathrm{mg})$ (Table 4$)$.

Adolescent consumers of potatoes in Case 2 (baked, boiled, mashed and mixtures, fried) as compared to non-consumers had higher $(p<0.05)$ intake of energy $(+11.5 \%)$, carbohydrate $(+9.33 \%)$, dietary fiber $(+6.47 \%)$, protein $(+8.94 \%)$, copper $(+8.74 \%)$, magnesium $(+5.81 \%)$, phosphorus $(+6.41 \%)$, potassium $(+16.8 \%)$, selenium $(+2.94 \%)$, sodium $(+10.6 \%)$, zinc $(+6.42 \%)$, niacin $(+11.2 \%)$, vitamin $B_{6}(+15.0 \%)$, vitamin $C(+8.91 \%)$, vitamin $\mathrm{E}(+5.72 \%)$, vitamin $\mathrm{K}(+9.26 \%)$, and total choline $(+11.3 \%)$; and lower $(p<0.05)$ intakes of vitamin A $(-4.62 \%)$ and folate $(-5.44 \%)$. Intakes of all other nutrients evaluated were similar $(p>0.05)$ among consumers and non-consumers of Case 2 potatoes (Table 5). 
Table 5. Energy and nutrients intake associated with potato consumption in adolescents (9-18 years of age, $n=16,633)$, NHANES 2001-2018; gender combined data.

\begin{tabular}{|c|c|c|c|c|c|c|}
\hline & \multicolumn{3}{|c|}{$\begin{array}{c}\text { Case } 2 \text { (Baked, Boiled, Mashed and Mixtures, } \\
\text { and Fried Potatoes) }\end{array}$} & \multicolumn{3}{|c|}{$\begin{array}{c}\text { Case } 3 \text { (Baked, Boiled, Mashed and Mixtures, } \\
\text { Fried Potatoes and Potato Chips) }\end{array}$} \\
\hline & Non-Consumers & Consumers & $p$ Value & Non-Consumers & Consumers & $p$ Value \\
\hline Energy (kcal) & $2017 \pm 13$ & $2249 \pm 17$ & $<0.0001$ & $1985 \pm 15$ & $2220 \pm 15$ & $<0.0001$ \\
\hline Carbohydrate (gm) & $268 \pm 2$ & $293 \pm 2$ & $<0.0001$ & $264 \pm 2$ & $290 \pm 2$ & $<0.0001$ \\
\hline Dietary fiber (gm) & $13.9 \pm 0.1$ & $14.8 \pm 0.2$ & $<0.0001$ & $13.9 \pm 0.2$ & $14.6 \pm 0.1$ & 0.0005 \\
\hline Protein (gm) & $72.7 \pm 0.6$ & $79.2 \pm 0.8$ & $<0.0001$ & $72.6 \pm 0.7$ & $77.7 \pm 0.7$ & $<0.0001$ \\
\hline Calcium (mg) & $1037 \pm 12$ & $1017 \pm 13$ & 0.2000 & $1038 \pm 13$ & $1021 \pm 11$ & 0.2560 \\
\hline Copper (mg) & $1.03 \pm 0.01$ & $1.12 \pm 0.01$ & $<0.0001$ & $1.02 \pm 0.01$ & $1.11 \pm 0.01$ & $<0.0001$ \\
\hline Iron (mg) & $15.3 \pm 0.2$ & $15.2 \pm 0.2$ & 0.8170 & $15.3 \pm 0.2$ & $15.2 \pm 0.1$ & 0.5838 \\
\hline Magnesium (mg) & $241 \pm 2$ & $255 \pm 3$ & $<0.0001$ & $239 \pm 2$ & $253 \pm 2$ & $<0.0001$ \\
\hline Phosphorus (mg) & $1295 \pm 11$ & $1378 \pm 13$ & $<0.0001$ & $1291 \pm 12$ & $1361 \pm 11$ & $<0.0001$ \\
\hline Potassium (mg) & $2124 \pm 18$ & $2480 \pm 25$ & $<0.0001$ & $2062 \pm 22$ & $2444 \pm 21$ & $<0.0001$ \\
\hline Selenium $(\mu \mathrm{g})$ & $102 \pm 1$ & $105 \pm 1$ & 0.0206 & $102 \pm 1$ & $105 \pm 1$ & 0.0504 \\
\hline Sodium (mg) & $3267 \pm 26$ & $3614 \pm 35$ & $<0.0001$ & $3233 \pm 32$ & $3559 \pm 31$ & $<0.0001$ \\
\hline Zinc (mg) & $10.9 \pm 0.1$ & $11.6 \pm 0.1$ & $<0.0001$ & $10.9 \pm 0.1$ & $11.5 \pm 0.1$ & 0.0002 \\
\hline Vitamin A (RE) $(\mu \mathrm{g})$ & $605.5 \pm 8.7$ & $577.9 \pm 9.6$ & 0.0174 & $613 \pm 10$ & $578 \pm 9$ & 0.0028 \\
\hline Thiamin (mg) & $1.66 \pm 0.02$ & $1.67 \pm 0.02$ & 0.7206 & $1.65 \pm 0.02$ & $1.67 \pm 0.02$ & 0.3245 \\
\hline Riboflavin (mg) & $2.09 \pm 0.02$ & $2.10 \pm 0.02$ & 0.7829 & $2.10 \pm 0.03$ & $2.09 \pm 0.02$ & 0.9084 \\
\hline Niacin (mg) & $22.4 \pm 0.2$ & $24.9 \pm 0.3$ & $<0.0001$ & $22.3 \pm 0.3$ & $24.5 \pm 0.2$ & $<0.0001$ \\
\hline Folate, DFE $(\mu \mathrm{g})$ & $570 \pm 7$ & $539 \pm 8$ & 0.0026 & $572 \pm 8$ & $544 \pm 7$ & 0.0044 \\
\hline Vitamin $\mathrm{B}_{6}(\mathrm{mg})$ & $1.73 \pm 0.02$ & $1.99 \pm 0.02$ & $<0.0001$ & $1.71 \pm 0.03$ & $1.95 \pm 0.02$ & $<0.0001$ \\
\hline Vitamin $B_{12}(\mu \mathrm{g})$ & $5.10 \pm 0.08$ & $5.21 \pm 0.07$ & 0.2476 & $5.12 \pm 0.09$ & $5.17 \pm 0.07$ & 0.6395 \\
\hline Vitamin C (mg) & $74.1 \pm 1.6$ & $80.7 \pm 1.9$ & 0.0033 & $72.1 \pm 1.6$ & $80.7 \pm 1.7$ & 0.0001 \\
\hline Vitamin D (D2 + D3) $(\mu \mathrm{g})$ & $5.45 \pm 0.10$ & $5.26 \pm 0.10$ & 0.1158 & $5.47 \pm 1.0$ & $5.29 \pm 0.09$ & 0.1232 \\
\hline Vitamin E (ATE) (mg) & $6.99 \pm 0.13$ & $7.39 \pm 0.09$ & 0.0116 & $6.60 \pm 0.16$ & $7.60 \pm 0.10$ & $<0.0001$ \\
\hline Vitamin K $(\mu \mathrm{g})$ & $64.8 \pm 1.7$ & $70.8 \pm 1.9$ & 0.0103 & $65.9 \pm 2.0$ & $68.6 \pm 1.6$ & 0.2785 \\
\hline Total choline (mg) & $256.2 \pm 3.0$ & $284.7 \pm 3.6$ & $<0.0001$ & $258 \pm 4$ & $277 \pm 3$ & 0.0001 \\
\hline
\end{tabular}

Data adjusted for age, gender and ethnicity; and presented as Least Square Mean \pm Standard Error. RE: retinol equivalent; DFE: dietary folate equivalent; ATE: alpha tocopherol equivalent.

Adolescent consumers of potatoes in Case 3 (baked, boiled, mashed and mixtures, fried, chips) as compared to non-consumers had higher $(p<0.01)$ intake of energy $(+11.8 \%)$, carbohydrate $(+9.85 \%)$, dietary fiber $(+5.04 \%)$, protein $(+7.02 \%)$, copper $(+8.82 \%)$, magnesium $(+5.86 \%)$, phosphorus $(+5.42 \%)$, potassium $(+18.5 \%)$, sodium $(+10.1 \%)$, zinc $(+5.50 \%)$, niacin $(+9.87 \%)$, vitamin $\mathrm{B}_{6}(+14.0 \%)$, vitamin $\mathrm{C}(+11.9 \%)$, vitamin $\mathrm{E}(+15.2 \%)$, and total choline $(+7.36 \%)$; and lower $(p<0.01)$ intakes of vitamin A $(-5.71 \%)$ and folate $(-4.90 \%)$. Intakes of all other nutrients evaluated were similar $(p>0.05)$ among consumers and non-consumers of Case 3 potatoes (Table 5).

Nutrient Adequacy: Compared to non-consumers, a lower $(p<0.05)$ proportion of adolescent consumers of potatoes in Case 1 (baked, boiled, mashed and mixtures) were below the EAR for carbohydrate, protein, copper, iron, magnesium, phosphorus, selenium, zinc, vitamin $\mathrm{A}$, thiamine, riboflavin, niacin, vitamin $\mathrm{B}_{6}$ and vitamin $\mathrm{C}$, and a higher $(p<0.05)$ proportion were above AI for potassium, sodium, and vitamin K (Table 6). 
Table 6. Nutrient inadequacy/adequacy in adolescent (9-18 years of age, $n=16,633$ ) potato consumers and non-consumers (NHANES 2001-2018, gender combined data).

\begin{tabular}{|c|c|c|c|c|c|c|c|c|c|}
\hline & \multicolumn{3}{|c|}{$\begin{array}{c}\text { Case } 1 \text { (Baked, Boiled, } \\
\text { and Mashed and Mixtures) }\end{array}$} & \multicolumn{3}{|c|}{$\begin{array}{l}\text { Case } 2 \text { (Baked, Boiled, Mashed and } \\
\text { Mixtures, and Fried Potatoes) }\end{array}$} & \multicolumn{3}{|c|}{$\begin{array}{c}\text { Case } 3 \text { (Baked, Boiled, Mashed } \\
\text { and Mixtures, Fried Potatoes, } \\
\text { and Potato Chips) }\end{array}$} \\
\hline & $\begin{array}{l}\text { Non- } \\
\text { Consumers }\end{array}$ & Consumers & $p$ Value & $\begin{array}{l}\text { Non- } \\
\text { Consumers }\end{array}$ & Consumers & $p$ Value & $\begin{array}{l}\text { Non- } \\
\text { Consumers }\end{array}$ & Consumers & $p$ Value \\
\hline & \multicolumn{9}{|c|}{ \% Population below Estimated Average Requirement (EAR) } \\
\hline Carbohydrate & $0.13 \pm 0.04$ & $\begin{array}{c}0.01 \pm \\
0.01\end{array}$ & 0.0026 & $0.18 \pm 0.06$ & $\begin{array}{c}0.03 \pm \\
0.01\end{array}$ & 0.0154 & $\begin{array}{c}0.22 \pm \\
0.08\end{array}$ & $\begin{array}{c}0.03 \pm \\
0.01\end{array}$ & 0.0206 \\
\hline Protein & $1.43 \pm 0.30$ & $\begin{array}{c}0.19 \pm \\
0.15\end{array}$ & 0.0002 & $1.85 \pm 0.45$ & $\begin{array}{c}0.41 \pm \\
0.14\end{array}$ & 0.0025 & $\begin{array}{c}1.98 \pm \\
0.56\end{array}$ & $\begin{array}{c}0.62 \pm \\
0.16\end{array}$ & 0.0203 \\
\hline Calcium & $61.9 \pm 1.0$ & $63.4 \pm 2.1$ & 0.5212 & $60.5 \pm 1.2$ & $\begin{array}{c}64.4 \pm \\
1.40\end{array}$ & 0.0313 & $60.1 \pm 1.3$ & $63.7 \pm 1.2$ & 0.0430 \\
\hline Copper & $7.16 \pm 0.65$ & $\begin{array}{c}1.46 \pm \\
0.42\end{array}$ & $<0.0001$ & $7.93 \pm 0.85$ & $\begin{array}{c}3.57 \pm \\
0.45\end{array}$ & $<0.0001$ & $\begin{array}{c}9.16 \pm \\
1.04\end{array}$ & $\begin{array}{c}3.90 \pm \\
0.38\end{array}$ & $<0.0001$ \\
\hline Iron & $4.01 \pm 0.37$ & $\begin{array}{c}2.65 \pm \\
0.36\end{array}$ & 0.0084 & $4.15 \pm 0.48$ & $\begin{array}{c}3.14 \pm \\
0.29\end{array}$ & 0.0726 & $\begin{array}{c}4.53 \pm \\
0.57\end{array}$ & $\begin{array}{c}3.16 \pm \\
0.28\end{array}$ & 0.0306 \\
\hline Magnesium & $59.5 \pm 0.8$ & $48.1 \pm 1.7$ & $<0.0001$ & $59.3 \pm 0.9$ & $\begin{array}{c}55.5 \pm \\
1.07\end{array}$ & 0.0071 & $59.9 \pm 1.1$ & $56.0 \pm 0.9$ & 0.0054 \\
\hline Phosphorus & $27.4 \pm 1.0$ & $18.8 \pm 2.5$ & 0.0011 & $29.3 \pm 1.2$ & $21.4 \pm 1.3$ & $<0.0001$ & $29.3 \pm 1.4$ & $\begin{array}{c}23.1 \pm \\
1.19\end{array}$ & 0.0006 \\
\hline Selenium & $0.40 \pm 0.11$ & $\begin{array}{c}0.04 \pm \\
0.06\end{array}$ & 0.0042 & $0.43 \pm 0.15$ & $\begin{array}{c}0.16 \pm \\
0.07\end{array}$ & 0.1003 & $\begin{array}{c}0.54 \pm \\
0.20\end{array}$ & $\begin{array}{c}0.17 \pm \\
0.06\end{array}$ & 0.0774 \\
\hline Zinc & $15.1 \pm 1.1$ & $8.8 \pm 1.6$ & 0.0015 & $16.8 \pm 1.3$ & $10.4 \pm 1.1$ & 0.0002 & $17.2 \pm 1.5$ & $11.5 \pm 1.1$ & 0.0023 \\
\hline Vitamin A & $41.5 \pm 1.1$ & $30.0 \pm 2.9$ & 0.0002 & $37.9 \pm 1.3$ & $42.0 \pm 1.5$ & 0.0461 & $37.3 \pm 1.5$ & $41.6 \pm 1.3$ & 0.0294 \\
\hline Thiamin & $3.64 \pm 0.51$ & $\begin{array}{c}1.10 \pm \\
0.37\end{array}$ & 0.0001 & $3.90 \pm 0.62$ & $\begin{array}{c}2.10 \pm \\
0.38\end{array}$ & 0.0137 & $\begin{array}{c}4.83 \pm \\
0.71\end{array}$ & $\begin{array}{c}1.99 \pm \\
0.37\end{array}$ & 0.0004 \\
\hline Riboflavin & $2.47 \pm 0.41$ & $\begin{array}{c}0.87 \pm \\
0.30\end{array}$ & 0.0017 & $2.82 \pm 0.50$ & $\begin{array}{c}1.41 \pm \\
0.32\end{array}$ & 0.0166 & $\begin{array}{c}2.81 \pm \\
0.61\end{array}$ & $\begin{array}{c}1.66 \pm \\
0.28\end{array}$ & 0.0850 \\
\hline Niacin & $1.08 \pm 0.27$ & $\begin{array}{c}0.11 \pm \\
0.08\end{array}$ & 0.0005 & $1.68 \pm 0.43$ & $\begin{array}{c}0.15 \pm \\
0.07\end{array}$ & 0.0005 & $\begin{array}{c}2.08 \pm \\
0.53\end{array}$ & $\begin{array}{c}0.24 \pm \\
0.08\end{array}$ & 0.0006 \\
\hline Folate, DFE & $7.42 \pm 0.84$ & $\begin{array}{l}5.75 \pm \\
1.12\end{array}$ & 0.2337 & $6.96 \pm 0.88$ & $\begin{array}{c}7.15 \pm \\
0.82\end{array}$ & 0.8771 & $\begin{array}{c}7.56 \pm \\
1.00\end{array}$ & $\begin{array}{c}6.82 \pm \\
0.78\end{array}$ & 0.5621 \\
\hline Vitamin $\mathrm{B}_{6}$ & $6.12 \pm 0.79$ & $\begin{array}{c}0.30 \pm \\
0.16\end{array}$ & $<0.0001$ & $7.85 \pm 1.09$ & $\begin{array}{c}1.37 \pm \\
0.35\end{array}$ & $<0.0001$ & $\begin{array}{c}8.80 \pm \\
1.29\end{array}$ & $\begin{array}{c}1.94 \pm \\
0.39\end{array}$ & $<0.0001$ \\
\hline Vitamin $\mathrm{B}_{12}$ & $2.58 \pm 0.45$ & $\begin{array}{c}1.37 \pm \\
0.44\end{array}$ & 0.0531 & $3.11 \pm 0.57$ & $\begin{array}{c}1.48 \pm \\
0.39\end{array}$ & 0.0174 & $\begin{array}{c}3.33 \pm \\
0.69\end{array}$ & $\begin{array}{c}1.72 \pm \\
0.35\end{array}$ & 0.0387 \\
\hline Vitamin C & $32.1 \pm 1.4$ & $17.2 \pm 2.2$ & $<0.0001$ & $31.6 \pm 1.7$ & $26.3 \pm 1.6$ & 0.0224 & $33.3 \pm 2.0$ & $26.3 \pm 1.5$ & 0.0058 \\
\hline Vitamin D & $91.9 \pm 0.6$ & $91.3 \pm 1.2$ & 0.6822 & $90.6 \pm 0.8$ & $93.1 \pm 0.8$ & 0.0270 & $90.3 \pm 0.9$ & $92.9 \pm 0.7$ & 0.0226 \\
\hline \multirow[t]{2}{*}{ Vitamin E } & $88.3 \pm 1.1$ & $88.5 \pm 2.1$ & 0.9446 & $87.6 \pm 1.3$ & $89.5 \pm 1.3$ & 0.3225 & $90.2 \pm 1.5$ & $87.3 \pm 1.3$ & 0.1381 \\
\hline & \multicolumn{9}{|c|}{ \% Population above Adequate Intake (AI) } \\
\hline $\begin{array}{l}\text { Dietary } \\
\text { fiber }\end{array}$ & $0.44 \pm 0.10$ & $\begin{array}{c}0.31 \pm \\
0.13\end{array}$ & 0.4215 & $0.57 \pm 0.13$ & $\begin{array}{c}0.26 \pm \\
0.08\end{array}$ & 0.0469 & $\begin{array}{c}0.65 \pm \\
0.18\end{array}$ & $\begin{array}{c}0.33 \pm \\
0.08\end{array}$ & 0.1037 \\
\hline Potassium & $28.3 \pm 1.0$ & $53.1 \pm 2.5$ & $<0.0001$ & $25.1 \pm 1.1$ & $42.4 \pm 1.5$ & $<0.0001$ & $22.7 \pm 1.2$ & $40.3 \pm 1.3$ & $<0.0001$ \\
\hline Sodium & $99.6 \pm 0.1$ & $100 \pm 0.04$ & 0.0006 & $99.5 \pm 0.2$ & $\begin{array}{c}99.9 \pm \\
0.03\end{array}$ & 0.0020 & $99.2 \pm 0.2$ & $\begin{array}{c}99.9 \pm \\
0.03\end{array}$ & 0.0013 \\
\hline Vitamin $\mathrm{K}$ & $37.2 \pm 1.6$ & $54.4 \pm 3.5$ & $<0.0001$ & $36.4 \pm 1.8$ & $46.2 \pm 2.2$ & 0.0007 & $37.0 \pm 2.2$ & $42.8 \pm 1.9$ & 0.0430 \\
\hline $\begin{array}{c}\text { Total } \\
\text { choline }\end{array}$ & $5.45 \pm 0.60$ & $\begin{array}{c}6.72 \pm \\
1.43\end{array}$ & 0.4122 & $5.96 \pm 0.76$ & $\begin{array}{c}5.25 \pm \\
0.80\end{array}$ & 0.5189 & $\begin{array}{c}6.35 \pm \\
0.91\end{array}$ & $\begin{array}{c}5.25 \pm \\
0.67\end{array}$ & 0.3302 \\
\hline
\end{tabular}

DFE: dietary folate equivalent.

Similarly, compared to non-consumers, a lower $(p<0.05)$ proportion of adolescent consumers of potatoes in Case 2 (baked, boiled, mashed and mixtures, fried) were below the EAR for carbohydrate, protein, copper, magnesium, phosphorus, zinc, thiamine, riboflavin, niacin, vitamin $\mathrm{B}_{6}$, vitamin $\mathrm{B}_{12}$ and vitamin $\mathrm{C}$; and a higher $(p<0.05)$ proportion were above AI for potassium, sodium, and vitamin $\mathrm{K}$. However, a higher $(p<0.05)$ proportion 
of consumers compared to non-consumers were below the EAR for calcium, vitamin A, and vitamin D; and a lower $(p<0.05)$ proportion were above AI for dietary fiber (Table 6).

Compared to non-consumers, a lower $(p<0.05)$ proportion of adolescent consumers of potatoes in Case 3 (baked, boiled, mashed and mixtures, fried, chips) also were below the EAR for carbohydrate, protein, copper, iron, magnesium, phosphorus, zinc, thiamine, niacin, vitamin $\mathrm{B}_{6}$, vitamin $\mathrm{B}_{12}$ and vitamin $\mathrm{C}$, and a higher $(p<0.05)$ proportion were above AI for potassium, sodium, and vitamin $\mathrm{K}$. However, a higher $(p<0.05)$ proportion of consumers compared to non-consumers were below the EAR for calcium, vitamin A, and vitamin $\mathrm{D}$ (Table 6).

\section{Discussion}

In the current cross-sectional analysis of data from nine cycles of NHANES (NHANES 2001-2018) using a nationally representative sample of over 16,000 US adolescents, potato consumption was associated with better diet quality, higher intake, and adequacy of several nutrients, including shortfall nutrients.

Potatoes are part of the starchy vegetables subgroup and are present in many different forms representing various cooking/processing methods in the diet. Potato chips, boiled potatoes, fries (French fries and home fries), and baked potatoes represent $28.7 \%, 23.5 \%$, $22.3 \%, 10.8 \%$, respectively, of starchy vegetables intake among children age $4-18$ years [36]. Limited data suggest that different forms of potatoes may have different nutritional and/or health attributes/outcomes [37-40]. For example, baked potatoes have a low glycemic index due to their high amount of resistant starch-a dietary fiber [41,42]. Potato chips are often considered as junk food, and fried potatoes may contain acrylamide, which is a potential carcinogen [43]. In the present analysis, we used three different definitions to define potato consumers-Case 1: those consuming baked, boiled, mashed, and mixtures; Case 2: those consuming baked, boiled, mashed and mixtures, fried; and Case 3: those consuming baked, boiled, mashed and mixtures, fried, chips and compared them to their respective non-consumers to differentiate specific attributes of different potato forms. Interestingly, irrespective of the potato consumer definition used, the consumers always had better diet quality, nutrient intake, and adequacy.

Potato consumers (by all three definitions) always had better diet quality, albeit in the $1.6-4.7 \%$ range) as assessed using HEI 2015. HEI is a validated measure of diet quality and is indicative of compliance/adherence of a person's diet to the eating pattern recommended by the Dietary Guidelines for Americans $[29,44]$ and is commonly used to evaluate diets and dietary interventions [45-47], food environments [45], to assess changes in the diet quality over time [46,47], and to validate other nutrition research tools and indexes [48]. It has also been used to understand relationships between nutrients/foods/dietary patterns and health-related outcomes in scientific studies [49-52]. With this metric, a higher score is indicative of compliance/adherence to dietary recommendations using 13 components (nine for adequacy and four for moderation), each of which relates to key recommendations of the 2015-2020 Dietary guidelines for Americans [29]. In the present analysis, potato consumers of baked, boiled, mashed and mixtures (Case 1) compared to non-consumers had a 2.1-point higher HEI 2015 total score and the score gradually increased with increasing intake quartiles. HEI 2015 total scores were also higher for potato consumers of baked, boiled, mashed and mixtures, fried (Case 2) and for consumers of baked, boiled, mashed and mixtures, fried, chips (Case 3 ) than their respective non-consumers; however, the difference was smaller ( +0.9 and +0.7 , respectively). Additionally, compared to Case 1 consumers, Case 2 and Case 3 consumers had more HEI 2015 subcomponent scores lower than non-consumers ( 2 subcomponents for Case 1 vs. 6 subcomponents for Case 2 and 7 subcomponents for Case 3) suggesting that diet quality for consumers was influences by other foods commonly consumed with fried potatoes and potato chips. However, in all cases, the total HEI score remained below 50 points (out of a possible 100) and indicates the need for broad improvement on many dietary quality components. 
Potato consumers had significantly higher intakes of dietary fiber, copper, magnesium, phosphorus, potassium, selenium, zinc, niacin, vitamin B6, vitamin C, vitamin E, vitamin $\mathrm{K}$ and total choline, and their intake increased with increasing potato intake quartiles. Additionally, potato consumers also had higher nutrient adequacy for copper, magnesium, phosphorus, potassium, zinc, thiamine, niacin, vitamin $B_{6}$, vitamin $B_{12}$, vitamin $C$, and vitamin $\mathrm{K}$ than non-consumers. Many of these nutrients are currently under-consumed, and especially dietary fiber and potassium are identified as "Dietary Components of Public Health Concern for Underconsumption" due to their inadequate intake [44]. Additionally, Dietary Guidelines for Americans, 2020-2025 [44] indicated that low intake of nutrient dense foods within food groups has led to low intakes of phosphorus, magnesium, and choline. Higher intakes of micronutrients among potato consumers, as observed in the present analysis, were also reported in earlier cross-sectional studies from both US and international cohorts $[19,20,26,53]$. Since potatoes are a good source of several of the above nutrients [2-4], potato consumption is naturally expected to lead to more nutrient dense diets and greater adequacy for nutrients.

Although potato consumers had higher dietary fiber intake than non-consumers, the difference was about $1 \mathrm{~g}$ or less and \% above $\mathrm{AI}$ remained very low (less than $1 \%$ population above AI). On the other hand, potato consumption was associated with a 300-400 mg/d increase in potassium and the \% of the population above the AI increased 17-25 percentage points. Similarly, potato consumption was also associated with significant and substantial increase in intake and population adequacy for copper, magnesium, phosphorus, vitamin $\mathrm{B}_{6}$, vitamin $\mathrm{C}$, and vitamin $\mathrm{K}$. However, potato consumers also had 130-230 more calories (kcal) than non-consumers. Substantially higher intake and higher adequacy of these nutrients among potato consumers indicates that regular inclusion of potatoes in the diet might be an effective strategy to improve the nutritional status of these nutrients.

However, potato consumption was also associated with increased sodium intake. While sodium is an essential nutrient, average intakes of sodium is high across US population compared to the Chronic Disease Risk Reduction Levels [44]. However, starchy vegetables, including potatoes, are a relatively minor source of sodium contributing to about $4 \%$ intake, compared to sandwiches or grain-based mixed dishes contributing to $21 \%$ and $8 \%$ intakes, respectively [44].

The strengths of this study include the use of a large nationally representative sample of adolescents achieved by combining several sets of NHANES data releases and the use of numerous covariates to adjust data to remove potential confounding; however, even with these covariates, some residual confounding may still exist. A major limitation of this study is the use of a cross-sectional study design, which cannot be used to determine cause and effect. The self-reported dietary recalls for dietary intake data relying on memory are potentially subject to reporting bias.

\section{Conclusions}

The results show that potato consumption was associated with better diet quality, higher nutrient intake, and improved nutrient adequacy among adolescents. Potatoes are nutrient rich foods and are a major contributor of starchy vegetables. Current intake of vegetables, including starchy vegetables, are less than the recommended levels [39]. Encouraging potato consumption, preferably without a lot of extra fat/sodium, may be an effective strategy for improving intakes and adequacy of vegetables and certain nutrients and achieving a healthier dietary pattern.

Author Contributions: The authors' responsibilities were as follows-S.A.: project conception, designed research, developed overall research plan, interpretation of the data, prepared the first draft of the manuscript, and revised the manuscript; V.L.F.III: project conception, designed research, developed overall research plan, NHANES database analysis and statistical analysis, interpretation of the data, and revised the manuscript. All authors have read and agreed to the published version of the manuscript. 
Funding: This research was funded by the Alliance for Potato Research \& Education (APRE). APRE had no role in analyses/interpretation of results and did not review the manuscript prior to submission.

Institutional Review Board Statement: The data used for this manuscript was from the National Health and Nutrition Examination Survey (NHANES) 2001-2018; data collection for NHANES was approved by the Research Ethics Review Board of the National Center for Health Statistics. NHANES has stringent consent protocols and procedures to ensure confidentiality and protection from identification. This study was a secondary data analysis, which lacked personal identifiers, therefore, did not require Institutional Review Board review.

Informed Consent Statement: The data used for this manuscript was from the National Health and Nutrition Examination Survey (NHANES) 2001-2018 and all participants or proxies provided written informed consent.

Data Availability Statement: The datasets analyzed in this study are available in the Center for Disease Control and Prevention repository; available online: http:/ / www.cdc.gov/nchs/nhanes/. (accessed on 11 December 2020).

Acknowledgments: The study and the writing of the manuscript were supported by Alliance for Potato Research \& Education (APRE).

Conflicts of Interest: S.A. as Principal of NutriScience LLC performs nutrition science consulting for various food and beverage companies and related entities. V.L.F.III as Senior Vice President of Nutrition Impact, LLC performs consulting and database analyses for various food and beverage companies and related entities.

\section{References}

1. Zaheer, K.; Akhtar, M.H. Potato production, usage, and nutrition-A review. Crit. Rev. Food Sci. Nutr. 2016, 56, 711-721. [CrossRef]

2. Beals, K.A. Potato nutrition and health. Am. J. Potato Res. 2019, 96, 102-110. [CrossRef]

3. Potato Nutrition Handbook. 2015. Available online: https://www.usapotatoes.com/uploads/file-downloads / files / potatonutrition-handbook-2015.pdf (accessed on 20 April 2021).

4. Camire, M.E.; Kubow, S.; Donnelly, D.J. Potatoes and human health. Crit. Rev. Food Sci. Nutr. 2009, 49, 823-840. [CrossRef] [PubMed]

5. Drewnowski, A. New metrics of affordable nutrition: Which vegetables provide the most nutrients for least cost. J. Acad. Nutr. Diet. 2013, 113, 1182-1187. [CrossRef]

6. USDA. Choose My Plate. Available online: https://www.choosemyplate.gov (accessed on 20 April 2021).

7. WHO. Diet, Nutrition and the Prevention of Chronic Diseases; WHO Tech. Rep. Ser. 916; WHO: Geneva, Switzerland, 2003 ; pp. 1-149.

8. NHS. The Eat Well Guide. Available online: https://www.nhs.uk/live-well/eat-well/the-eatwell-guide/ (accessed on 20 April 2021).

9. Kim, S.A.; Moore, L.V.; Galuska, D.; Wright, A.P.; Harris, D.; Grummer-Strawn, L.M.; Merlo, C.L.; Nihiser, A.J.; Rhodes, D.G. Vital Signs: Fruit and vegetable intake among children-United States, 2003-2010. MMWR 2014, 63, 671-676.

10. What We Eat in America, NHANES 2009-2010, Individuals 2 Years and over (Excluding Breast-Fed Children), Day 1 Dietary Intake Data, Weighted. FPED 2009-2010. Available online: https://www.ars.usda.gov/ARSUserFiles/80400530/pdf/fped/ Table_1_FPED_GEN_0910.pdf (accessed on 20 April 2021).

11. Anderson, G.H.; Soeandy, C.D.; Smith, C.E. White vegetables: Glycemia and satiety. Adv. Nutr. 2013, 4, 356S-367S. [CrossRef]

12. Birt, D.F.; Boylston, T.; Hendrich, S.; Jane, J.L.; Hollis, J.; Li, L.; McClelland, J.; Moore, S.; Phillips, G.J.; Rowling, M.; et al. Resistant starch: Promise for improving human health. Adv. Nutr. 2013, 4, 587-601. [CrossRef] [PubMed]

13. Keenan, M.J.; Zhou, J.; Hegsted, M.; Pelkman, C.; Durham, H.A.; Coulon, D.B.; Martin, R.J. Role of resistant starch in improving gut health, adiposity, and insulin resistance. Adv. Nutr. 2015, 6, 198-205. [CrossRef] [PubMed]

14. Muraki, I.; Rimm, E.B.; Willett, W.C.; Manson, J.E.; Hu, F.B.; Sun, Q. Potato consumption and risk of type 2 diabetes: Results from three prospective cohort studies. Diabetes Care 2016, 39, 376-384. [CrossRef] [PubMed]

15. Borgi, L.; Rimm, E.B.; Willett, W.C.; Forman, J.P. Potato intake and incidence of hypertension: Results from three prospective US cohort studies. BMJ 2016, 353, i2351. [CrossRef]

16. Borch, D.; Juul-Hindsgaul, N.; Veller, M.; Astrup, A.; Jaskolowski, J.; Raben, A. Potatoes and risk of obesity, type 2 diabetes, and cardiovascular disease in apparently healthy adults: A systematic review of clinical intervention and observational studies. Am. J. Clin. Nutr. 2016, 104, 489-498. [CrossRef] [PubMed]

17. Mazidi, M.; Katsiki, N.; Mikhailidis, D.P.; Pella, D.; Banach, M. Potato consumption is associated with total and cause-specific mortality: A population-based cohort study and pooling of prospective studies with 98,569 participants. Arch. Med. Sci. 2020, 16, 260-272. [CrossRef] 
18. Oslo, L.E.W.; Fernandes, M.M. Relationship of white potato to other vegetable consumption by schoolchildren and adolescents in the USA: National Health and Nutrition Examination Survey, 2003-2008. Public Health Nutr. 2013, 16, $1933-1936$.

19. Freedman, M.R.; Keast, D.R. White potatoes, including french fries, contribute shortfall nutrients to children's and adolescents' diets. Nutr. Res. 2011, 31, 270-277. [CrossRef]

20. Nicklas, T.A.; Liu, Y.; Islam, N.; O’Neil, C.E. Removing potatoes from children's diets may compromise potassium intake. Adv. Nutr. 2016, 7, 247S-253S. [CrossRef] [PubMed]

21. Institute of Medicine. School Meals: Building Blocks for Healthy Children; The National Academies Press: Washington, DC, USA, 2010.

22. Food and Nutrition Service (FNS); USDA. Nutrition standards in the National School Lunch and School Breakfast Programs. Final rule. Fed. Register. 2012, 77, 4088-40167.

23. Healthy, Hunger-Free Kids Act of 2010; Public Law 111-296, 124 Stat. 3183. 2010. Available online: https://www.govinfo.gov/ content/pkg/PLAW-111publ296/pdf/PLAW-111publ296.pdf (accessed on 17 May 2021).

24. Institute of Medicine. WIC Food Packages: Time for a Change; National Academies Press: Washington, DC, USA, 2006.

25. McGuire, S. Institute of Medicine. Review of WIC food packages: An evaluation of white potatoes in the cash value voucher: Letter report. Washington (DC): The National Academies Press, 2015. Adv. Nutr. 2015, 6, 863-864. [CrossRef] [PubMed]

26. Yiannakou, I.; Yuan, M.; Pickering, R.T.; Singer, M.R.; Moore, L.L. Potato consumption is not associated with elevated cardiometabolic risk in adolescent girls. Curr. Dev. Nutr. 2020, 4, 1506. [CrossRef]

27. Centers for Disease Control and Prevention (CDC); National Center for Health Statistics. National Health and Nutrition Examination Survey. National Center for Health Statistics: Hyattsville, MD, USA. Available online: https://www.cdc.gov/nchs/ nhanes/index.htm (accessed on 11 December 2020).

28. Krebs-Smith, S.M.; Pannucci, T.E.; Subar, A.F.; Kirkpatrick, S.I.; Lerman, J.L.; Tooze, J.A.; Wilson, M.M.; Reedy, J. Update of the Healthy Eating Index: HEI-2015. J. Acad. Nutr. Diet. 2018, 118, 1591-1602. [CrossRef]

29. U.S. Department of Health and Human Services; U.S. Department of Agriculture. 2015-2020 Dietary Guidelines for Americans, 8th ed.December 2015. Available online: http:/ / health.gov/dietaryguidelines/2015/guidelines/ (accessed on 17 May 2021).

30. Haytowitz, D.B.; Ahuja, J.K.C.; Wu, X.; Somanchi, M.; Nickle, M.; Nguyen, Q.A.; Roseland, J.M.; Williams, J.R.; Patterson, K.Y.; Li, Y.; et al. USDA National Nutrient Database for Standard Reference, Legacy Release. Nutrient Data Laboratory, Beltsville Human Nutrition Research Center, ARS, USDA. Available online: https:/ / data.nal.usda.gov / dataset/usda-national-nutrient-databasestandard-reference-legacy-release (accessed on 11 December 2020).

31. USDA/ARS. USDA Food and Nutrient Database for Dietary Studies. Food Surveys Research Group Home Page. Available online: http:/ / www.ars.usda.gov/nea/bhnrc/fsrg (accessed on 11 December 2020).

32. USDA MyPyramid Equivalents Database. Food Surveys Research Group. Available online: https://www.ars.usda.gov/ northeast-area/beltsville-md-bhnrc/beltsville-human-nutrition-research-center/food-surveys-research-group/docs / mpeddatabases-for-downloading/ (accessed on 11 December 2020).

33. USDA/ARS. USDA Food Patterns Equivalents Database. Food Surveys Research Group. Available online: https://www. ars.usda.gov / northeast-area/beltsville-md-bhnrc/beltsville-human-nutrition-research-center/food-surveys-research-group / docs/fped-overview/ (accessed on 11 December 2020).

34. Tooze, J.A.; Kipnis, V.; Buckman, D.W.; Carroll, R.J.; Freedman, L.S.; Guenther, P.M.; Krebs-Smith, S.M.; Subar, A.F.; Dodd, K.W. A mixed-effects model approach for estimating the distribution of usual intake of nutrients: The NCI method. Stat. Med. 2010, 29, 2857-2868. [CrossRef] [PubMed]

35. Institute of Medicine. DRIs: Applications in Dietary Assessment; National Academies Press: Washington, DC, USA, 2000.

36. 2020 Dietary Guidelines Advisory Committee; Food Pattern Modeling Team. Added Sugars: Food Pattern Modeling: Ages 2 Years and Older. 2020 Dietary Guidelines Advisory Committee Project; U.S. Department of Agriculture: Washington, DC, USA, 2020.

37. Palazoglu, T.K.; Savran, D.; Gokmen, V. Effect of cooking method (baking compared with frying) on acrylamide level of potato chips. J. Food Sci. 2010, 75, E25-E29. [CrossRef]

38. Foot, R.J.; Haase, N.U.; Grob, K.; Gonde, P. Acrylamide in fried and roasted potato products: A review on progress in mitigation. Food Addit. Contam. 2007, 24, 37-46. [CrossRef] [PubMed]

39. Soh, N.L.; Brand-Miller, J. The glycaemic index of potatoes: The effect of variety, cooking method and maturity. Eur. J. Clin. Nutr. 1999, 53, 249-254. [CrossRef] [PubMed]

40. Leeman, M.; Ostman, E.; Bjorck, I. Glycaemic and satiating properties of potato products. Eur. J. Clin. Nutr. 2008, 62, 87-95. [CrossRef] [PubMed]

41. Raatz, S.K.; Idso, L.; Johnson, L.K.; Jackson, M.I.; Combs, G.F., Jr. Resistant starch analysis of commonly consumed potatoes: Content varies by cooking method and service temperature but not by variety. Food Chem. 2016, 208, 297-300. [CrossRef] [PubMed]

42. Yang, Y.; Achaerandio, I.; Pujola, M. Effect of the intensity of cooking methods on the nutritional and physical properties of potato tubers. Food Chem. 2016, 197 Pt B, 1301-1310. [CrossRef]

43. Virk-Baker, M.K.; Nagy, T.R.; Barnes, S.; Groopman, J. Dietary acrylamide and human cancer: A systematic review of literature. Nutr. Cancer 2014, 66, 774-790. [CrossRef]

44. U.S. Department of Agriculture; U.S. Department of Health and Human Services. Dietary Guidelines for Americans, 2020-2025, 9th ed.December 2020. Available online: https:/ / DietaryGuidelines.gov (accessed on 17 May 2021). 
45. Reedy, J.; Krebs-Smith, S.M.; Bosire, C. Evaluating the food environment: Application of the Healthy Eating Index-2005. Am. J. Prev. Med. 2010, 38, 465-471. [CrossRef]

46. Hiza, H.A.; Casavale, K.O.; Guenther, P.M.; Davis, C.A. Diet quality of Americans differs by age, sex, race/ethnicity, income, and education level. J. Acad. Nutr. Diet. 2013, 113, 297-306. [CrossRef]

47. Juan, W.Y.; Guenther, P.M.; Kott, P.S. Diet Quality of Older Americans in 1994-96 and 2001-02 as Measured by the Healthy Eating Index-2005. Nutrition Insight 41; United States Department of Agriculture Center for Nutrition Policy and Promotion: Alexandria, VA, USA, 2008.

48. Fulgoni, V.L.; Keast, D.R.; Drewnowski, A. Development and validation of the Nutrient-rich Foods Index: A tool to measure nutritional quality of foods. J. Nutr. 2009, 139, 1549-1554. [CrossRef]

49. Nicklas, T.A.; O'Neil, C.E.; Fulgoni, V.L. Diet quality is inversely related to cardiovascular risk factors in adults. J. Nutr. 2012, 142, 2112-2118. [CrossRef] [PubMed]

50. Chiuve, S.E.; Fung, T.T.; Rimm, E.B.; Hu, F.B.; McCullough, M.L.; Wang, M.; Stampfer, M.J.; Willett, W.C. Alternative dietary indices both strongly predict risk of chronic disease. J. Nutr. 2012, 142, 1009-1018. [CrossRef] [PubMed]

51. Reedy, J.; Mitrou, P.N.; Krebs-Smith, S.M.; Wirfält, E.; Flood, A.; Kipnis, V.; Leitzmann, M.; Mouw, T.; Hollenbeck, A.; Schatzkin, A.; et al. Index-based dietary patterns and risk of colorectal cancer: The NIH-AARP Diet and Health Study. Am. J. Epidemiol. 2008, 168, 38-48. [CrossRef] [PubMed]

52. O'Neil, C.E.; Nicklas, T.A.; Rampersaud, G.C.; Fulgoni, V.L. One hundred percent orange juice consumption is associated with better diet quality, improved nutrient adequacy, and no increased risk for overweight/obesity in children. Nutr. Res. 2011, 31, 673-682. [CrossRef] [PubMed]

53. Gibson, S.; Kurilich, A.C. The nutritional value of potatoes and potato products in the UK diet. Nutr. Bull. 2013, 38, 389-399. [CrossRef] 